\title{
The economic burden of schizophrenia
}

\author{
Linda M. Davies (correspondence); and Michael F. DRummond, Health Services \\ Management Centre, University of Birmingham, Park House, 40 Edgbaston Park \\ Road, Birmingham B15 2RT
}

Schizophrenia is an expensive disease. In the USA, it affects approximately $1 \%$ of the population but patients occupy $25 \%$ of all hospital beds. In Australia, schizophrenia was found to affect less than $10 \%$ of the number experiencing a myocardial infarction and yet the costs of medical services for treating schizophrenia are $75 \%$ of those for myocardial infarction.

In view of these statistics, we decided to estimate the economic impact of schizophrenia in the UK. The economic implications of the disease extend beyond the use of health and personal social services to its morbidity and mortality implications as well as its impact on the quality of life of patients and their families.

\section{Incidence and prevalence}

The incidence rates reported in the literature ranged from $0.11 / 1000$ population to $0.25 / 1000$ population (all ages). The mean average of these rates is $0.172 /$ 1000 population (s.d. $=0.05$ ), and the median is 0.18 / 1000 population. The reported prevalence rates showed a similar variation, from $2.1 / 1000$ population to $3.4 / 1000$ population (all ages) and $2.5 / 1000$ population to $6.8 / 1000$ population (aged $15+$ ).

The estimated UK population for 1987 was 56.9 million (all ages) and 46.1 million (aged 15 years and over). Therefore the estimated annual incidence and prevalence of schizophrenia for the UK in 1987 ranges from approximately 6,000 to 14,000 people (mean 10,000, s.d. 3,500, midpoint 10,200). The prevalence of the disease ranges from 115,000 to 313,000 people (mean 185,000 , s.d. 93,000 , midpoint $214,000)$.

\section{Demographic characteristics of people with schizophrenia}

The age of onset of schizophrenia tends to be higher for women than for men. The median age of onset has been estimated at 29 years for men compared to 37 years for women.

The distribution of people with schizophrenia between socio-economic class differed to that of the general population. Approximately $36 \%$ of schizophrenia patients were from classes I, II and III, 30\% from classes IV and V and $35 \%$ were students, unemployed or housewives (e.g. Johnstone et al, 1986). In contrast, $67 \%$ of the general population were in socio-economic classes I, II and III, $30 \%$ in classes IV and $\mathrm{V}$ and $7 \%$ were classed as unemployed.

\section{Mortality and morbidity from schizophrenia}

The average life expectancy of men with schizophrenia is slightly lower than would be expected for the population as a whole. The life expectancy of men with schizophrenia is approximately 37 years (using the median age at onset) compared to 43 years for men without schizophrenia. One cause of mortality is suicide. Prudo \& Blum (1987) found that $5 \%$ of patients with chronic schizophrenia successfully committed suicide compared to $0.8 \%$ of the general population.

Schizophrenia is also associated with chronic longterm disability. The disease can be divided into three stages of illness: mental health problems prior to the episode of diagnosis or first admission to mental health facilities, the episode of diagnosis or first admission, and further episodes of illness. A proportion of people with schizophrenia never recover from the first episode of illness.

Sartorius et al (1986) found that about $80 \%$ of patients had been ill for up to six months prior to diagnosis or first hospital admission for schizophrenia. The remaining $20 \%$ had been ill for over six months.

A large proportion of people will be admitted on an in-patient basis to mental illness hospitals or units at the onset of schizophrenia. In 1986, there were 3,871 first admissions for schizophrenia in England. This represented approximately $50 \%$ of the estimated incidence of schizophrenia in England for 1986. However, the number of first admissions to mental illness hospitals or units for schizophrenia in England has shown a steady decline from 5,413 in 1976 to 3,871 in 1986 . The average length of stay for first admission has been reported at 8.2 weeks (median, Johnstone et al, 1986).

The pattern of illness following the first episode varies. Sartorius et al (1986) estimate that up to $40 \%$ of patients will have one brief episode followed by 
remission with or without residual symptoms. A further $20 \%$ will have an intermediate outcome characterised by further episodes of illness, with symptom-free intervals and no evidence of personality deterioration. The remaining $40 \%$ of patients will have a severe or chronic outcome of continuing illness and conspicuous personality changes.

Prudo \& Blum (1987) found that 5 years after entry to an International Pilot Study of Schizophrenia, $19 \%$ had no further episodes of illness, $30 \%$ had further episodes which lasted in total less than 1 year, $10 \%$ had further episodes and had been ill for $1-2.5$ years and $41 \%$ had further episodes resulting in illness for over 2.5 years. Of this latter group $7 \%$ of patients had died, and $10 \%$ had never recovered from the episode of inclusion to the study.

On the basis of these results, between $50 \%$ and $60 \%$ of people with schizophrenia could be considered to have a long-standing and/or limiting illness. This compared with approximately $33 \%$ of the general population reporting a long-standing and/or limiting illness.

\section{Costs of schizophrenia}

The costs to society of schizophrenia comprise three main categories: direct treatment costs, indirect costs of lost production, and intangibles. The direct treatment costs include hospital in-patient and outpatient care, other residential care, community based services (e.g. social worker, community psychiatric nurse, GP) and drug therapy.

\section{Total annual costs of schizophrenia}

One way of estimating the economic burden of disease is to calculate the total cost for a given year. This is known as the prevalence approach. The direct treatment costs of schizophrenia are presented in Table I. The average cost per person per year is approximately $£ 1,670$. The major cost items are hospital in-patient care ( $£ 572)$, other residential care (£662) and day care (£228). The total annual costs of treatment and care in the UK can be estimated by multiplying the average cost per person by the average treated prevalence of schizophrenia. This gives a cost of approximately $£ 310$ million (1987 prices) to the health and personal social services. Hospital and residential care costs were the major items of expenditure and represented $74 \%$ of total direct costs. Non-residential care costs (excluding drug therapy) were $23 \%$ of total costs, and the costs of drug therapy represented $3 \%$ of the total direct costs. These annual direct treatment costs of schizophrenia represent $1.6 \%$ of total health and personal social services expenditure in the UK in 1987.

In addition to the direct treatment costs, $70-80 \%$ of patients are likely to be unemployed, absent from
TABLE I

Average annual resource use and costs per person: UK 1987

\begin{tabular}{lr}
\hline Service & $\begin{array}{c}\text { Average cost/ } \\
\text { per person }\end{array}$ \\
\hline Hospital in-patient stay (days) & 572.45 \\
Other residential care (days) & 661.60 \\
Out-patient (visits) & 56.15 \\
Daycare (days) & 228.33 \\
Community psychiatric nurse (visits) & 10.17 \\
Social worker (visits) & 11.07 \\
GP (visits) & 41.79 \\
Depot injection clinic (visits) & 32.43 \\
Drug therapy & 55.82 \\
Total & $1,669.81$ \\
\hline
\end{tabular}

work or unable to do housework each year, resulting in costs of lost production. However, some of these people would probably have been unemployed in any case. In order to calculate the indirect costs of schizophrenia it was assumed that $20 \%$ of patients (excluding housewives) would not have been in paid employment if they had not suffered from schizophrenia (this corresponds to the average proportion of the general population not in paid employment (OPCS, 1989). Under these assumptions approximately 111,000 people with schizophrenia are estimated to incur production losses due to the disease only. The additional assumption was made that these people would be unemployed for the full year. The national average wage in 1987 was $£ 11,650$ p.a. (Central Statistical Office, 1989), giving a total indirect morbidity cost due to schizophrenia of $£ 1,296$ million per year.

In addition to the indirect morbidity costs there would also be indirect costs of premature mortality. However, there was insufficient evidence about the average mortality rate in the total schizophrenic population to calculate the costs of lost production with any certainty. The monetary direct and indirect costs are therefore in the region of $£ 1,606$ million per year. This estimate is based on the mean average treated prevalence of schizophrenia in the UK. High and low cost estimates can be generated using different prevalence rates in a sensitivity analysis, to give the possible range of costs. Using the lowest and highest estimate of prevalence, the total annual direct treatment costs range from $£ 192$ million to $£ 523$ million. The indirect costs range from $£ 805$ million (assumes 69,120 people unable to work) to $£ 2,191$ million (assumes 188,100 people unable to work). The range of total direct and indirect costs is $£ 997$ million to $£ 2,714$ million per year using 1987 prices. 


\section{Lifetime costs of schizophrenia}

An alternative method of calculating the costs of schizophrenia is to calculate the lifetime costs of a cohort of people with schizophrenia from onset of the disease to death, by likely outcome of the disease. This is known as the incidence approach. It was assumed that there would be four likely outcomes, based on the classification of symptomatic outcome used by Prudo \& Blum (1987).

An outcome of 1 assumes that patients will only have 1 episode of illness, with an average duration of 22 weeks. An outcome of 2 assumes that patients have further episodes of illness lasting less than 1 year in total, outcome 3 is for patients who have more than 1 episode and are ill for 1-2.5 years (mean 1.75 years) and outcome 4 refers to patients who have more than 1 episode and are ill for more than 2.5 years. For this latter group it was assumed for the purposes of estimating costs, that all these patients would require some form of treatment and care for the remainder of their lives. Of these $75 \%$ ( $31 \%$ of the cohort) would receive predominantly community based care (group $4 a)$ and $25 \%$ ( $10 \%$ of the cohort) would be long-stay residents in mental illness hospitals or units, hostels or group homes (group 4b).

The lifetime direct treatment costs for the cohort are approximately $£ 305$ million (discounted value*). The costs varied substantially between the different outcome groups. The lifetime costs for outcome group 1 were $£ 2.5$ million, for outcome group 2 they were $£ 4.5$ million, for outcome group 3 they were £2.9 million and for outcome group 4(a) the lifetime direct treatment costs were $£ 53.4$ million. For the group of patients assumed to receive long-term residential care (4(b)), the lifetime direct treatment costs were estimated at $£ 241.8$ million. The patients in groups $4(a)$ and $4(b)$ were assumed to be $41 \%$ of the cohort. However, this group accounted for $97 \%$ of the total lifetime direct treatment costs.

Furthermore, the cohort would incur substantial indirect (morbidity) costs in terms of lost production through unemployment due to illness. To calculate the indirect costs, it was assumed that outcome group 1 would be unemployed or unable to work for 22 weeks, outcome group 2 would be unemployed or unable to work for 1 year, and the remaining groups would be unemployed or unable to work for the remainder of their lives (using a life expectancy of 37 years). Finally it was assumed that $20 \%$ of patients would have been unemployed if they did not have schizophrenia.

The discounted lifetime costs of lost production were estimated at $£ 9.1$ million for outcome group 1 , $£ 32.3$ million for outcome group $2, £ 168.2$ million for

* Discounting is a procedure by which costs and benefits occurring in the future are compared, in an economic evaluation, with those occurring in the present.
TABLE II

Lifetime costs of treatment/care and lost production at different rates of incidence: UK 1987 (Es millions)

\begin{tabular}{lccccc}
\hline Incidence & 1 & 2 & 3 & $4 a$ & $4 b$ \\
\hline $\begin{array}{l}6,260 \\
\text { (low) }\end{array}$ & 7.4 & 23.5 & 109.3 & 367.2 & 262.0 \\
$\begin{array}{l}9,800 \\
\text { (mean) }\end{array}$ & 11.6 & 36.8 & 171.1 & 575.0 & 410.0 \\
$\begin{array}{l}14,200 \\
\text { (high) }\end{array}$ & 16.9 & 53.4 & 248.0 & 833.1 & 594.2 \\
\hline
\end{tabular}

outcome group $3, £ 521.6$ million for outcome group 4 (a) and $f 168.2$ million for the long-stay patients in group $4(\mathrm{~b})$. The total lifetime indirect costs for the cohort were therefore $£ 899.4$ million. The total direct and indirect costs were $£ 1204.4$ million.

The accuracy of these costs depends on the incidence rate assumed, and the average resource use and costs for each outcome group. High and low estimates of the incidence of schizophrenia were used to calculate the range of possible costs. However, there was insufficient evidence to calculate high and low estimates of resource use. The lifetime costs using low $(6,260)$ and high $(14,200)$ estimates of incidence are given in Table II. The total discounted direct and indirect costs using the low incidence value were $£ 769.4$ million and using the high incidence value were $£ 1745.6$ million.

\section{Comment}

This paper has shown that schizophrenia, in common with other chronic long-term illnesses, represents a major economic burden to society. From the public policy viewpoint, the estimates of the annual treatment cost in health care resources give an indication of the budgetary commitment that will be required in this and future years. The estimates of lifetime cost give a baseline against which new treatment interventions can be assessed.

New treatment interventions are critical, since demonstrating that a given illness has a large economic burden is a necessary, but not sufficient condition, for further action. In addition, new, costeffective treatments to combat the disease need to be developed.

The treatment and care of people with schizophrenia has changed from predominantly hospital in-patient based care to community based care with in-patient admissions when necessary. Between 1976 and 1986 the proportion of first admissions to 
mental illness hospitals or units for schizophrenia declined from approximately $70 \%(5,413)$ to $46 \%$ $(3,871)$.

The proportion of all people with schizophrenia admitted to mental illness hospitals or units declined from about $21 \%$ to $16 \%$ over the same period (DOH 1988). Furthermore the proportion of people with an in-patient admission of less than 1 month increased from $9.6 \%$ in 1975 to $22 \%$ in 1981 . The proportion of people with an in-patient admission of over 5 years declined from $4 \%$ in 1975 to $2.6 \%$ in 1981. The number of readmissions in 1971 was 26,245 compared to 24,571 in 1981 . The majority of people with schizophrenia now live in their own homes or community based residential accommodation, using day care or out-patient services. Approximately $75 \%$ of people receive regular neuroleptic medication which represents $0.6 \%$ of the total economic cost of schizophrenia. Up to $75 \%$ of people with chronic schizophrenia are maintained in the community.

The development of adequate community care services and medicines that will enable patients to be maintained in the community is therefore a priority if the economic burden of schizophrenia is to be reduced.

\section{References}

Central Statistical Office (1989) Social Trends, 19. London: HMSO.

DePARTMENT OF HeAlth (1988) Health and Personal Social Services Statistics for England. London: HMSO.

Johnstone, E. C., Crow, T. J., Johnson, A. L. \& MacMillan, J. F. (1986) The Northwick Park study of first episodes of schizophrenia. I. Presentation of the illness and problems relating to admission. British Journal of Psychiatry, 148, 115-120.

Prudo, R. \& Blum, H. M. (1987) Five year outcome and prognosis in schizophrenia. British Journal of Psychiatry, 150, 345-354.

Sartorius, N., Jablensky, A., Korten, A., ERnberg, G., ANKER, M., COOPER, J. E. \& DAY, R. (1986) Early manifestations and first contact incidence of schizophrenia in different cultures: a preliminary report on the initial evaluation phase of the WHO collaborative study on determinants of outcome of severe mental disorders. Psychological Medicine, 16, 909-928.

A fuller list of references and data sources is available from the authors.

\section{Reduced subscriptions}

The International Journal of Psycho-Analysis and the International Review of Psycho-Analysis are offering subscriptions at a reduced rate to members of organisations wishing to subscribe through their societies as block subscribers: $£ 39$ for Journal, $£ 36$ for Review,
$£ 68$ for combined subscription. The appropriate amount should be sent to Mrs Jean Wales at the College (cheque made payable to the Royal College of Psychiatrists) not later than 12 November 1990. 